\title{
NLO leptoquark production and decay: the narrow-width approximation and beyond
}

\section{J.B. Hammett and D.A. Ross}

School of Physics and Astronomy, University of Southampton, Highfield, Southampton SO17 1BJ, U.K.

E-mail: jh12g10@soton.ac.uk, doug@soton.ac.uk

ABSTRACT: We study the leptoquark model of Buchmüller, Rückl and Wyler, focusing on a particular type of scalar $\left(R_{2}\right)$ and vector $\left(U_{1}\right)$ leptoquark. The primary aim is to perform the calculations for leptoquark production and decay at next-to-leading order (NLO) to establish the importance of the NLO contributions and, in particular, to determine how effective the narrow-width-approximation (NWA) is at NLO. For both the scalar and vector leptoquarks it is found that the NLO contributions are large, with the larger corrections occurring for the case vector leptoquarks. For the scalar leptoquark it is found that the NWA provides a good approximation for determining the resonant peak, however the NWA is not as effective for the vector leptoquark. For both the scalar and vector leptoquarks there are large contributions away from the resonant peak, which are missing from the NWA results, and these make a significant difference to the total cross-section.

Keywords: NLO Computations, Hadronic Colliders

ARXIV EPRINT: 1501.06719 


\section{Contents}

1 Introduction 1

2 Scalar leptoquarks $\quad 3$

2.1 NWA results 5

2.2 Non-factorisable results 8

2.3 Renormalization/factorisation scale dependence for scalar leptoquarks $\quad 10$

3 Vector leptoquarks $\quad 11$

3.1 NWA results $\quad 12$

$\begin{array}{lll}3.2 & \text { Non-factorisable results } & 15\end{array}$

3.3 Renormalization/factorization scale dependence for vector leptoquarks $\quad 16$

$\begin{array}{llr}4 & \text { Conclusions } & 17\end{array}$

$\begin{array}{lll}4.1 & \text { Scalar leptoquarks } & 17\end{array}$

$\begin{array}{lll}4.2 & \text { Vector leptoquarks } & 18\end{array}$

\section{Introduction}

For the purpose of this paper will use the leptoquark model of W. Buchmüller, R. Rückl and D. Wyler [1] — a model which conserves lepton and baryon number thereby avoiding the problem of proton decay. The search for leptoquarks is an important consideration for the ATLAS [2-4] and CMS experiments [5-7] and the lepton and baryon number conservation feature of the Buchmüller, Rückl and Wyler model allows us to consider a leptoquark mass which is accessible to these LHC experiments.

A leptoquark production process can produce either a single leptoquark or a pair of leptoquarks, and in the study by Belyaev et al. [8] it has been shown that pair production dominates for low leptoquark masses $\left(m_{\mathrm{LQ}} \sim 100 \mathrm{GeV}\right)$, but as the mass is increased single leptoquark production becomes the more dominant contribution (see also [9] and [10]). For this reason we are focusing on single leptoquark production and the primary objective of this paper is to study single leptoquark production and decay at next-to-leading (NLO), in particular to determine the effectiveness of the narrow-width approximation (NWA) at NLO. ${ }^{1}$ In the case of scalar leptoquarks (within the NWA) it was found that electroweak corrections are negligible compared to the QCD corrections and therefore for the purpose of this study only QCD corrections will be considered for the NLO calculations.

The key assumptions of the NWA are that the leptoquark process can be factorised into a production and decay process (where the decay-width is much smaller than the leptoquark

\footnotetext{
${ }^{1}$ The cross-section for leptoquark pair production at the LHC and Tevatron have been studied by Kramer et al. $[11,12]$.
} 
mass) and that the interference between the different helicity states for the production and decay processes is negligible. With these assumptions ${ }^{2}$ the leptoquark production and decay can be calculated as separate processes involving an on-shell leptoquark.

In addition to these assumptions, the studies by Kauer et al. [13-15] identify other factors which need to be considered when using the NWA. These include the effect of non-factorisable contributions and the break-down of the NWA when the decay involves a decay product with a mass that approaches that of the parent particle. The effect of nonfactorisable contributions will be relevant to this paper, however the second consideration does not affect leptoquark production in the NWA since the leptoquark decay products are assumed to be massless in the high energy limit.

The NLO calculations are performed numerically using FORTRAN with the virtual corrections calculated using SAMURAI [16] and the cancellation of the infrared divergences implemented using the dipole subtraction method [17, 18]. In calculating the virtual corrections it was found that some of the loop contributions introduced numerical instabilities requiring the calculations to be performed using quadruple precision within FORTRAN.

The Buchmüller, Rückl and Wyler model includes both scalar and vector leptoquarks and these fall into two categories: those with fermion number ${ }^{3}|F|=0$ and those with fermion number $|F|=2$. In this model the effective Lagrangian for the interactions of leptoquarks with leptons and quarks is

$$
\mathcal{L}_{\text {quark+lepton }}=\mathcal{L}_{|F|=2}+\mathcal{L}_{|F|=0}
$$

with

$$
\begin{aligned}
\mathcal{L}_{|F|=2}= & \left(g_{1 L} \bar{q}_{L}^{c} i \tau_{2} l_{L}+g_{1 R} \bar{u}_{R}^{c} e_{R}\right) S_{1}+\tilde{g}_{1 R} \bar{d}_{R}^{c} e_{R} \tilde{S}_{1}+g_{3 L} \bar{q}_{L}^{c} i \tau_{2} \tau l_{L} S_{3} \\
& +\left(g_{2 L} \bar{d}_{R}^{c} \gamma^{\mu} l_{L}+g_{2 R} \bar{q}_{L}^{c} \gamma^{\mu} e_{R}\right) V_{2 \mu}+\tilde{g}_{2 L} \bar{u}_{R}^{c} \gamma^{\mu} l_{L} \tilde{V}_{2 \mu}+\text { c.c. }
\end{aligned}
$$

and

$$
\begin{aligned}
\mathcal{L}_{|F|=0}= & \left(h_{2 L} \bar{u}_{R} l_{L}+h_{2 R} \bar{q}_{L} i \tau_{2} e_{R}\right) R_{2}+\tilde{h}_{2 L} \bar{d}_{R} l_{L} \tilde{R}_{2} \\
& +\left(h_{1 L} \bar{q}_{L} \gamma^{\mu} l_{L}+h_{1 R} \bar{d}_{R} \gamma^{\mu} e_{R}\right) U_{1 \mu}+\tilde{h}_{1 R} \bar{u}_{R} \gamma^{\mu} e_{R} \tilde{U}_{1 \mu} \\
& +h_{3 L} \bar{q}_{L} \tau \gamma^{\mu} l_{L} U_{3 \mu}+\text { c.c. }
\end{aligned}
$$

where $q_{L}$ and $l_{L}$ are the left-handed quark and lepton doublets respectively and $e_{R}, u_{R}$, $d_{R}$ are the right handed charged leptons, $\mathrm{u}$ and d-quarks respectively.

In this paper we will start by considering the $R_{2}$ scalar leptoquark, which has fermion number $|F|=0$ and is an isospin doublet with electric charges $+5 / 3$ and $+2 / 3$ and couples to the quark and lepton sector with couplings $h_{2 L}$ and $h_{2 R}-$ as detailed in eq. (1.3). The leptoquark production and decay process will first be considered within the NWA and the results compared against the full non-factorisable process to determine the effectiveness of the NWA. This procedure will then be repeated with the $U_{1}$ vector leptoquark. The $U_{1}$

\footnotetext{
${ }^{2}$ Note: the second assumption only applies to vector leptoquarks, which have different helicity states.

${ }^{3}$ Fermion number $F$ is defined as $F=3 B+L$ where $B$ and $L$ are the baryon and lepton numbers respectively.
} 

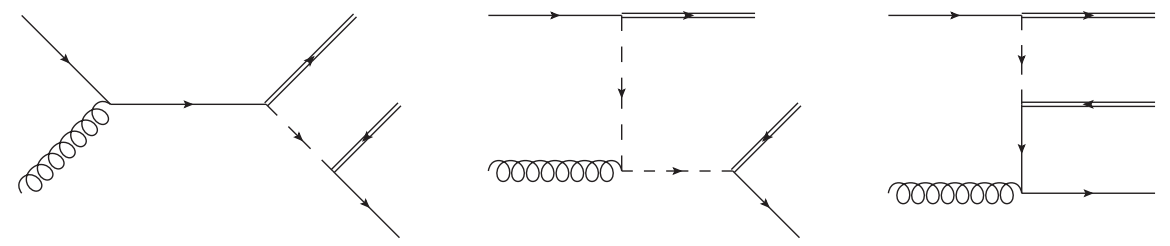

Figure 1. The LO contributions to the core process for leptoquark production. The first two diagrams can be factorised into leptoquark production and decay amplitudes whereas the third diagram is non-factorisable.

leptoquark also has fermion number $|F|=0$ and is an isospin singlet with electric charge $+2 / 3$ and couples to the quarks and leptons with couplings $h_{1 L}$ and $h_{1 R}-$ also shown in eq. (1.3). Having considered both scalar and vector leptoquarks the paper will conclude with a discussion and comparison between the scalar and vector leptoquark results.

In addition to studying the effectiveness of the NWA at NLO this paper will also look at the sensitivity of the NLO results to the renormalisation and factorisation scales.

In performing these calculations the center-of-mass energy has been set to $\sqrt{s}=$ $14 \mathrm{TeV}$. The leptoquark mass has been chosen to be $m_{\mathrm{LQ}}=750 \mathrm{GeV}$ and the couplings have been set to $\underbrace{h_{1 L}=h_{1 R}}_{U_{1} \text { couplings }}=\underbrace{h_{2 L}=h_{2 R}}_{R_{2} \text { couplings }}=1.07$ (i.e. taken from $\alpha_{s}$ evaluated at $m_{\mathrm{LQ}}$ ). The renormalisation and factorisation scales $\mu$ and $\mu_{F}$ have been set to $\mu=\mu_{F}=m_{\mathrm{LQ}}$.

In section 2 we consider the case of scalar leptoquarks and vector leptoquarks in section 3. In section 4 we present our conclusions.

\section{Scalar leptoquarks}

For the study of scalar leptoquarks we will consider the $R_{2}$ type leptoquark as described in [1]. The core process being studied is

$$
u+g \rightarrow e^{-}+e^{+}+u
$$

and the LO Feynman diagrams contributing to this process are shown in figure 1.

When studying leptoquark production at NLO there are three main contributions to the final result. Firstly there are the LO order contributions which come from the diagrams shown figure 1, then there are the NLO virtual and real QCD corrections to this process - with example diagrams shown in figures 2 and 3. Finally at NLO there are additional initial state contributions to the leptoquark production process such as the quark-quark initial state shown in figure 4. There are similar contributions from quark-antiquark, antiquark-antiquark, and gluon-gluon scattering. At the order of perturbation theory of the NLO corrections to the core process (2.1) these are given by tree-level amplitudes with an additional final-state parton. Some of the additional NLO calculations suffer from initial state singularities. There is no corresponding virtual correction with a cancelling infrared divergence - the divergences are absorbed into the parton distribution functions (PDF) and in keeping with our treatment of the infrared singularities in the NLO corrections to the core process, they are handled using the dipole subtraction method $[17,18]$. 

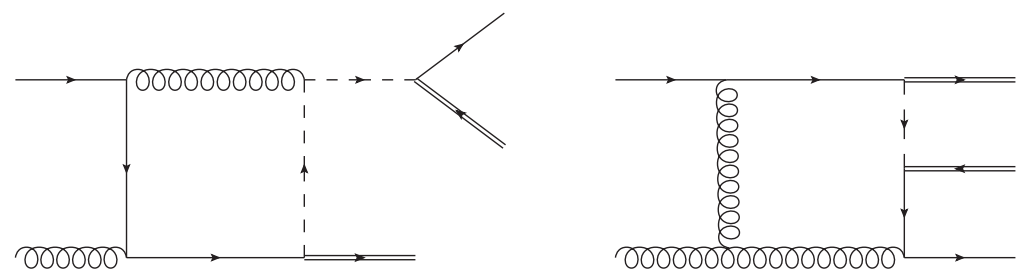

Figure 2. Example virtual corrections to the leptoquark production and decay process. The box diagram on the left is an example of a factorisable process whereas the pentagon diagram on the right is non-factorisable.
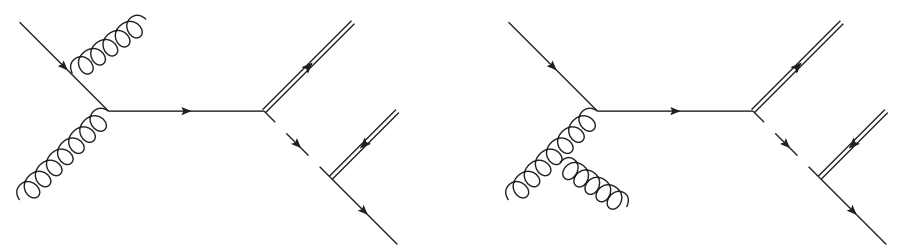

Figure 3. Example real corrections to the leptoquark production and decay process. The interference between these two diagrams contributes to the cancellation of the infrared divergences arising from the virtual corrections.

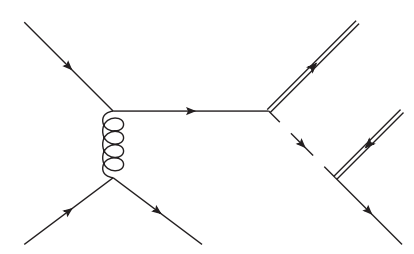

Figure 4. Example of an additional NLO contribution. This process shows leptoquark production from a quark-quark initial state.

In addition to the above mentioned contributions there are also background diagrams to consider. The main background to this process arises from Drell-Yan pair production in the presence of an accompanying jet. The cross-section for this process is considerably smaller than the total Drell-Yan cross-section. Furthermore, away from the resonant region for the leptoquark we expect the relative contribution from this process to be negligible as it does not contribute to a resonance in the invariant mass of the lepton-jet system. Since this process involves the emission of an intermediate $Z$ or photon, we expect that even away from the resonance region, the cross-section will be suppressed by the fourth power ratio of the electroweak coupling to the coupling of the leptoquark, which we take to be of the order of the strong coupling. The amplitude for the Drell-Yan process rapidly decreases with increasing invariant mass of the lepton pair (above the $Z$-mass resonance) and so any remaining background interference can be eliminated by imposing an appropriate minimum cut on the lepton-pair invariant mass. For this reason the contributions from the additional background diagrams have been excluded from this study.

All of the results in this paper show the differential cross-section versus the invariant mass, $m_{\text {inv }}$, of the jet + anti-lepton system evaluated over the range $\left[0,2 m_{\mathrm{LQ}}\right]$. In the 


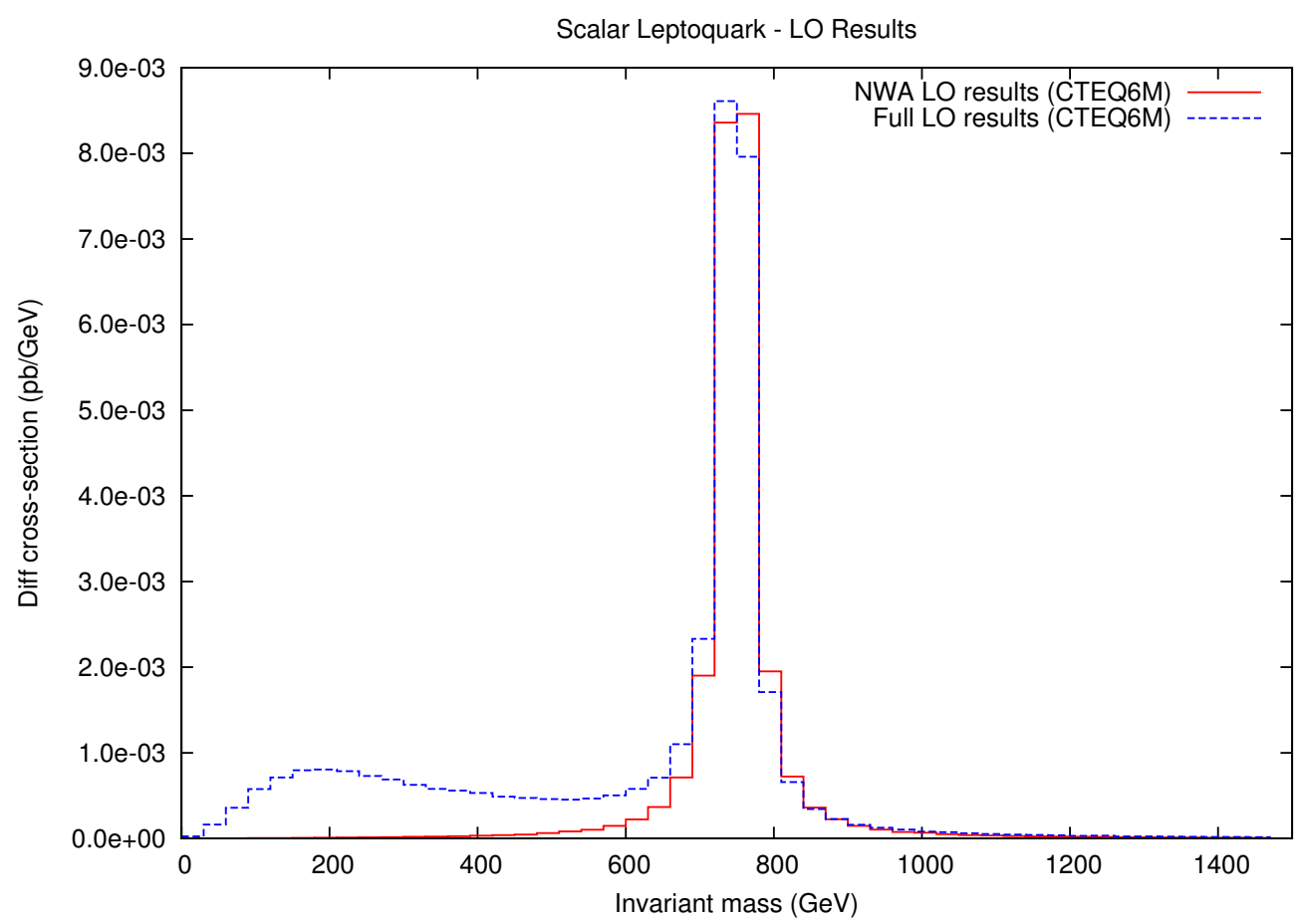

Figure 5. LO results for producing a scalar leptoquark - comparing the NWA to the full nonfactorisable process (CTEQ6M). The dashed line includes the effect of the non-factorising graph at LO.

case of one jet the invariant mass is defined as $m_{\mathrm{inv}}^{2} \equiv\left(p_{e^{+}}+p_{\mathrm{jet}}\right)^{2}$ where $p_{e^{+}}$is the 4 momentum of the anti-lepton and $p_{\text {jet }}$ is the 4-momentum of the jet. When there are two jets in the final state there are two such invariant masses, $m_{\text {inv }}{ }^{(1)}$ and $m_{\text {inv }}{ }^{(2)}$ where $m_{\text {inv }^{(i)}}^{2} \equiv\left(p_{e^{+}}+p_{\text {jet }_{i}}\right)^{2}, \quad i=1,2$. When constructing the differential cross-section w.r.t. $m_{\text {inv }}$ we include in a given bin of $m_{\text {inv }}$ any event in which either of the invariant masses lies within that bin. As it is not possible to distinguish experimentally between the two jets, in order to determine which jet arises from the decay of a leptoquark, an event is deemed to lie within a certain bin if it contains a positron and any jet with invariant mass within that bin. In the resonant region, the contribution from the "wrong" jet (i.e. that which did not arise from the leptoquark decay) will make a very small relative contribution to the differential cross-section.

\section{$2.1 \quad$ NWA results}

Looking first at the NWA results, figure 5 shows the LO contribution. As can be seen from the plot the NWA provides a symmetrical resonance with a peak near $m_{\mathrm{LQ}}=750 \mathrm{GeV}$ with a height of $8.46 \times 10^{-3} \mathrm{pb} / \mathrm{GeV}$. The total cross-section at LO is $0.74 \mathrm{pb}$.

Referring to figure 6 the NLO QCD corrections to the core parton process, (2.1), make a significant contribution to the process. There is still a symmetric resonant peak, but now with a height of $1.23 \times 10^{-2} \mathrm{pb} / \mathrm{GeV}$ which gives an enhancement of $45 \%$ over the LO result. For the QCD corrections the total cross-section is $1.07 \mathrm{pb}$ giving an increase of $45 \%$ 


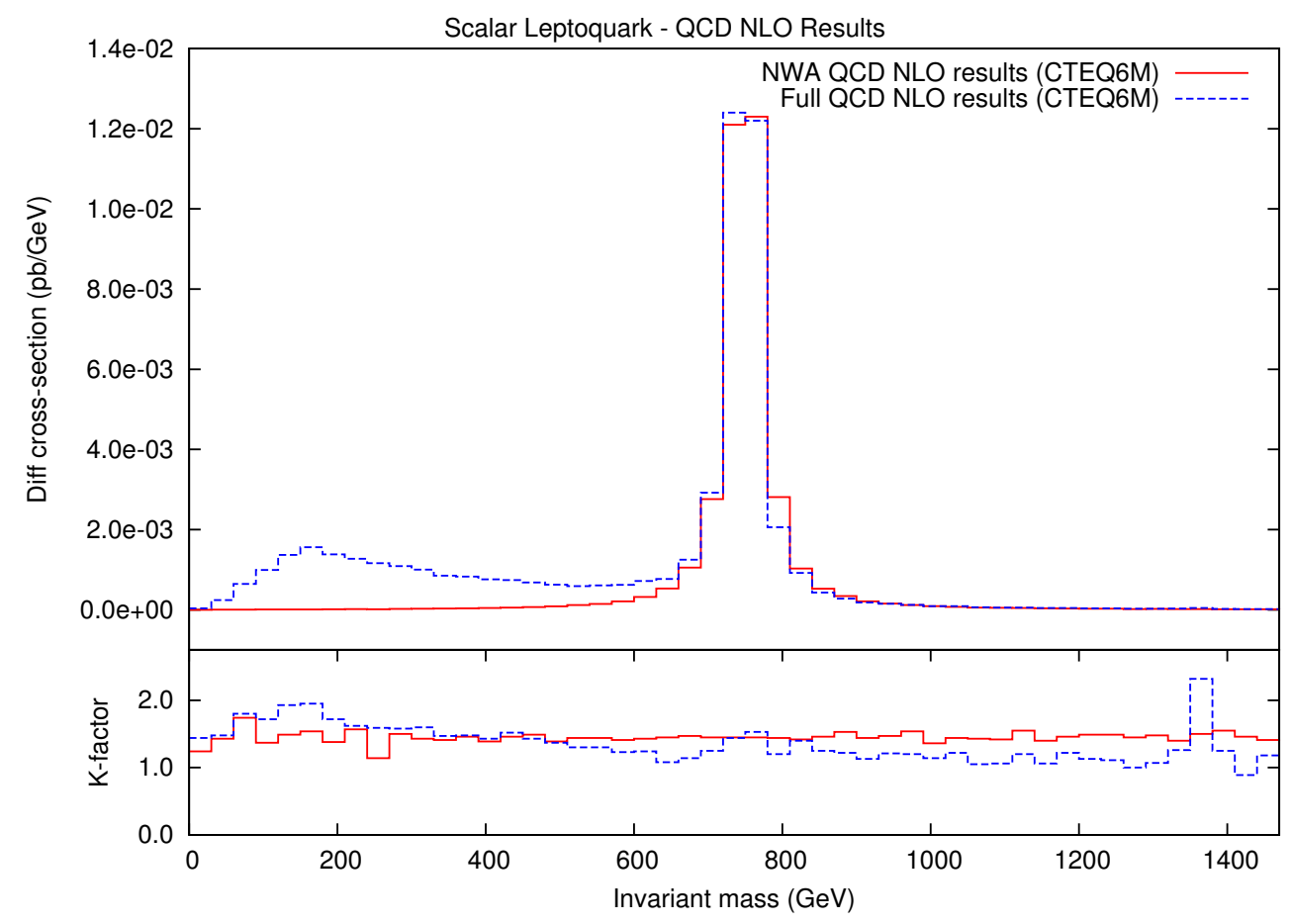

Figure 6. NLO results for producing a scalar leptoquark from the core partonic process - comparing the NWA to the full non-factorisable process (CTEQ6M). These results include the QCD corrections only.

over the LO result. The K-factor for the NLO QCD corrections is reasonably constant across the invariant mass distribution with an average value $\sim 1.5$

Finally the contributions from the additional partonic processes are shown in figure 7 . As discussed in section 2 the additional partonic processes are the NLO tree-level amplitudes which involve different initial state partons such as the quark-quark initial state shown in figure 4 . These additional contributions also enhance the LO process. The height of the resonant peak is $9.57 \times 10^{-3} \mathrm{pb} / \mathrm{GeV}$ giving an enhancement of $13 \%$ over the LO result. The total cross-section is $0.84 \mathrm{pb}$ which gives an increase over the LO result of $14 \%$. The K-factor for the additional partonic processes is $\sim 1.0$ across the invariant mass distribution, but does show an increased K-factor for low values of the invariant mass.

The combined results are shown in figure 8 and give the total NLO contributions to the leptoquark production process in the NWA. The total NLO contributions give a resonant peak height of $1.34 \times 10^{-2} \mathrm{pb} / \mathrm{GeV}$ adding a sizeable enhancement of $58 \%$ to the LO result. The total cross-section at NLO is $1.17 \mathrm{pb}$ which gives a $59 \%$ increase over the LO result. ${ }^{4}$ The K-factor for the total NLO contributions is $\sim 1.5$ across the invariant mass distribution and shows an increased $\mathrm{K}$-factor for low values of the invariant mass coming from the additional partonic processes. These results are summarised in tables 1 and 2 .

\footnotetext{
${ }^{4}$ Similar large corrections to single leptoquark production have been reported by Plehn et al. [19] and by Alves .et .al. [20].
} 


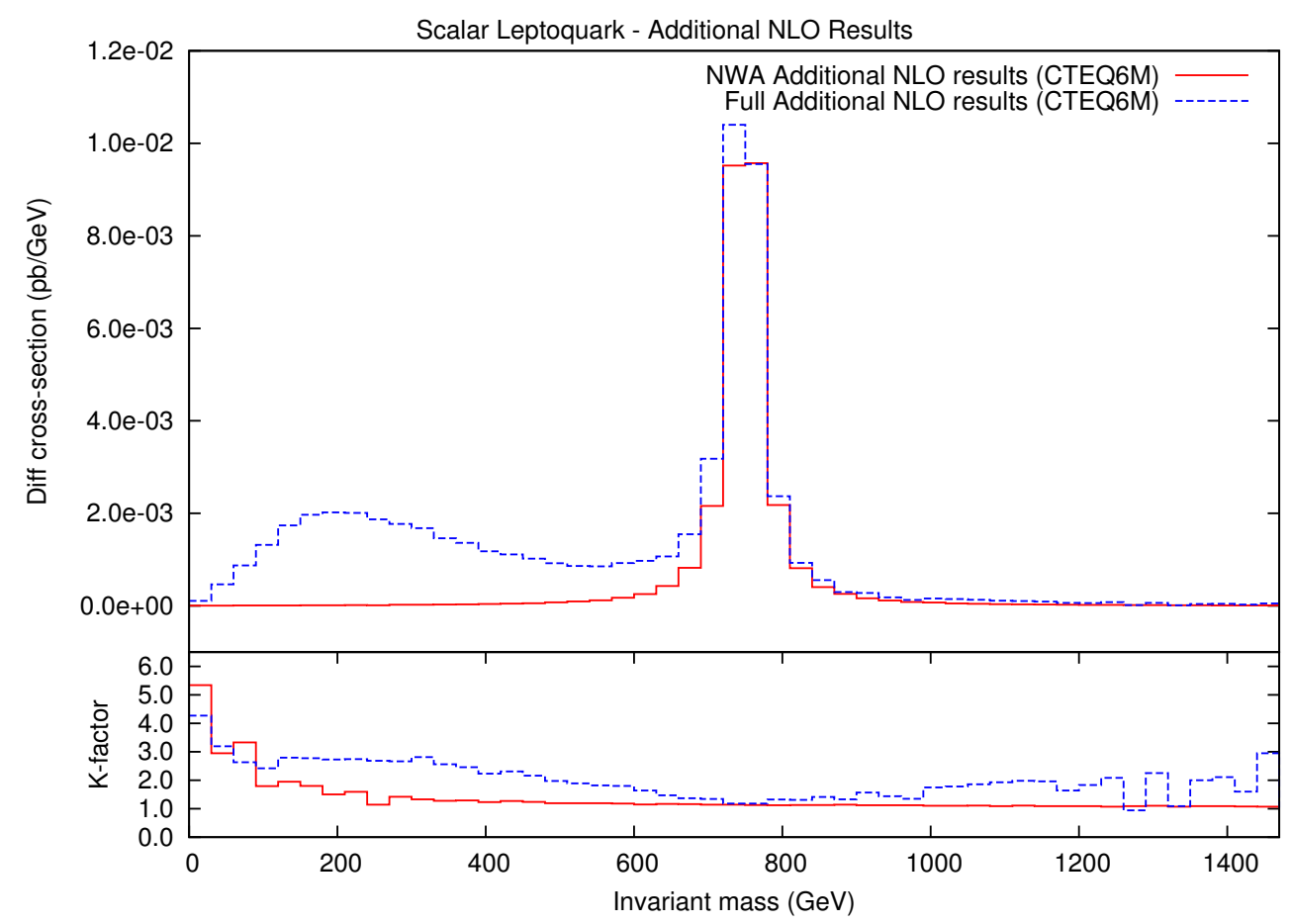

Figure 7. Additional NLO results (i.e. from the additional partonic processes) for producing a scalar leptoquark - comparing the NWA to the full non-factorisable process (CTEQ6M). These results include QCD corrections only.

\begin{tabular}{|l|r|r|}
\hline Correction Type & Peak Height $(\mathrm{pb} / \mathrm{GeV})$ & Percentage change on LO \\
\hline LO & $8.46 \times 10^{-3}$ & - \\
\hline NLO QCD & $1.23 \times 10^{-2}$ & $+45 \%$ \\
\hline NLO Additional & $9.57 \times 10^{-3}$ & $+13 \%$ \\
\hline NLO Total & $1.34 \times 10^{-2}$ & $+58 \%$ \\
\hline
\end{tabular}

Table 1. Summary of the CTEQ6M scalar results in the NWA.

\begin{tabular}{|l|r|r|}
\hline Correction Type & Total cross-section $(\mathrm{pb})$ & Percentage change on LO \\
\hline LO & 0.74 & - \\
\hline NLO QCD & 1.07 & $+45 \%$ \\
\hline NLO Additional & 0.84 & $+14 \%$ \\
\hline NLO Total & 1.17 & $+59 \%$ \\
\hline
\end{tabular}

Table 2. Summary of the CTEQ6M scalar results in the NWA. 


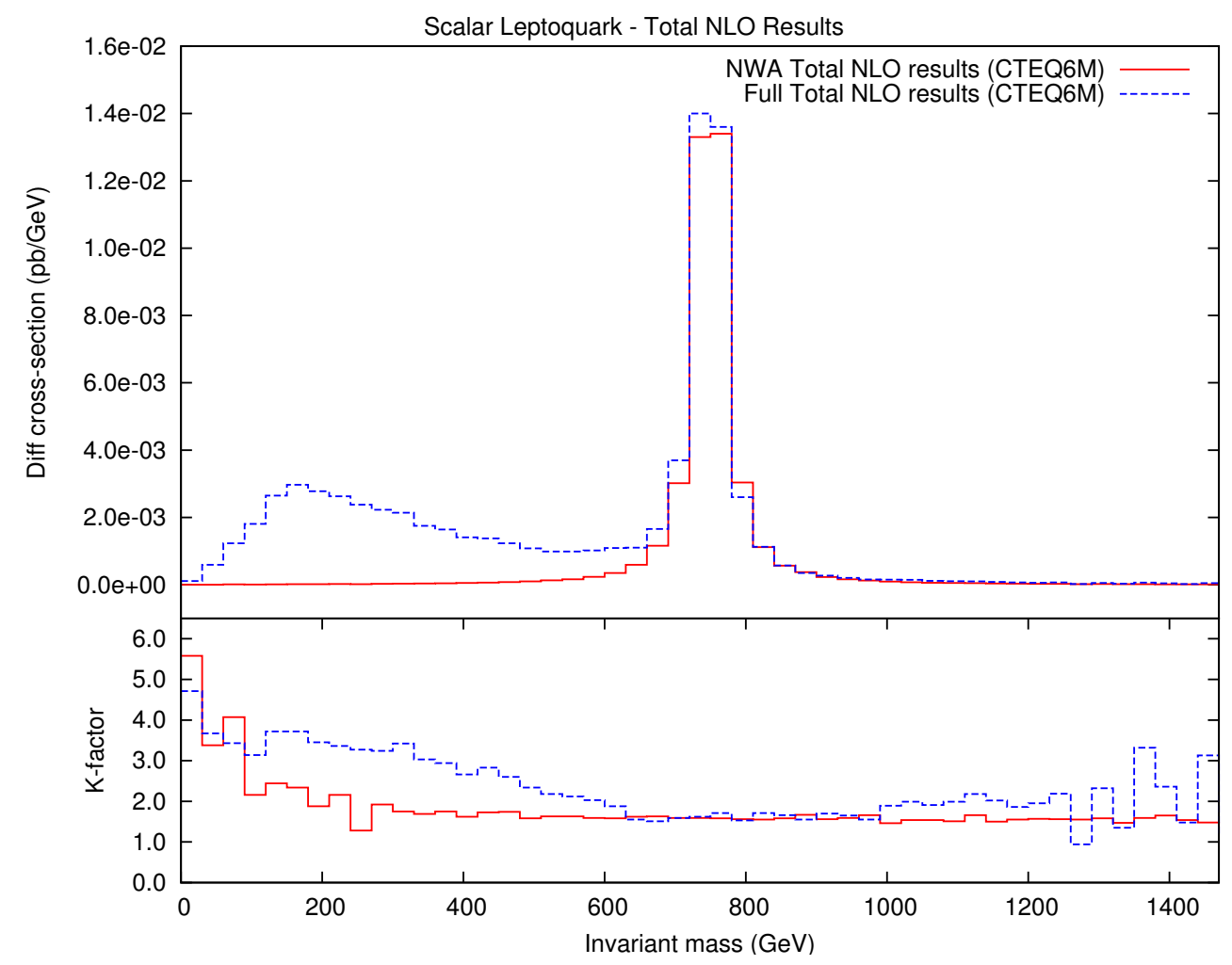

Figure 8. Total NLO results for producing a scalar leptoquark - comparing the NWA to the full non-factorisable process (CTEQ6M).

\subsection{Non-factorisable results}

Figure 5 shows the contribution of the LO non-factorizing graph of figure 1. In common with the NWA result the main feature in the distribution is the resonance with a peak near $m_{\mathrm{LQ}}=750 \mathrm{GeV}$. This has a peak height of $8.61 \times 10^{-3} \mathrm{pb} / \mathrm{GeV}$ which is very close to the NWA result. The key difference between the inclusion of the non-factorizing graph and the NWA result is that the distribution is no longer symmetric around the peak and we see that the non-factorisable contributions give an enhancement to the distribution for values of the invariant mass $m_{\mathrm{inv}}<m_{\mathrm{LQ}}$. The total cross-section at $\mathrm{LO}$ is $1.08 \mathrm{pb}$ in comparison to the NWA the total cross-section is larger. This is due to the enhancement to the distribution away from the resonance.

The results for the QCD corrections to the core process, (2.1), are shown in figure 6 and give a similar distribution to the LO results, with an enhancement to the resonant peak. The peak height is increased by $44 \%$ to $1.24 \times 10^{-2} \mathrm{pb} / \mathrm{GeV}$ and comparing this with the NWA peak the peaks are again very close. For the QCD corrections the total cross-section is $1.56 \mathrm{pb}$ which again is larger than the cross-section in the NWA and gives an enhancement over the LO cross-section of $45 \%$. The K-factor for the QCD contributions is reasonably constant over the invariant mass distribution, but shows more variation than the NWA result.

The contributions at NLO from the additional partonic processes are shown in figure 7. This also gives a similar distribution to the LO result, but in addition to an enhancement 


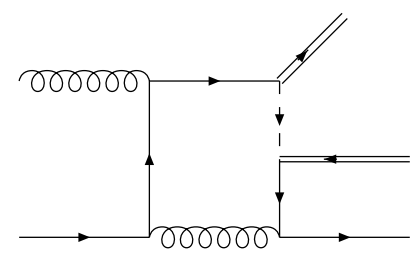

(a)

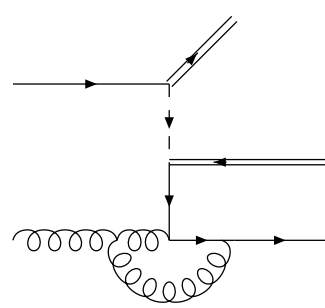

(b)

Figure 9. Graph (a) is an example of a non-factorizing virtual correction in which one end of the internal gluon is attached to an incoming parton whereas the other end is attached to one of the leptoquark decay products. NLO. Graph (b) is a virtual correction to the additional contribution present at tree-level beyond the NWA in which a leptoquark is exchanged in the $t$-channel.

to the resonant peak there is also a further enhancement in the region $m_{\text {inv }}<m_{\mathrm{LQ}}$. The resonant peak has a height of $1.02 \times 10^{-2} \mathrm{pb} / \mathrm{GeV}$ giving an enhancement over the LO result of $18 \%$. Again comparing the peak height with the peak in the NWA the peak heights are close. The total cross-section with the additional NLO results is $1.75 \mathrm{pb}$ and is considerably larger than the cross-section from NWA. The K-factor for the additional partonic processes shows more variation than the NWA result. As with the NWA results there is an increase in the $\mathrm{K}$-factor for both low and high values of the invariant mass distribution.

Combining the results gives the total NLO contribution to the leptoquark process and is shown in figure 8. The height of the resonant peak is $1.40 \times 10^{-2}$ which gives an enhancement of $62 \%$ compared with the LO peak. There is also a further enhancement in the region $m_{\text {inv }}<m_{\mathrm{LQ}}$ which is primarily due to the contributions from additional partonic processes at NLO. The cross-section for the total NLO result is $2.24 \mathrm{pb}$ which is an increase of $107 \%$ over the LO result, this is primarily due to the additional NLO contributions. The $\mathrm{K}$-factor for the total NLO contributions coincides with the K-factor for the NWA result around the resonant peak, but shows an increase (with some fluctuation) at both low and high values of the invariant mass distribution. A summary of these results is shown in tables 3 and 2 .

At NLO there is considerable enhancement, partly due to non-factorising one-loop corrections in which a virtual gluon is exchanged between incoming and outgoing partons (an example of which is shown in figure 9(a)) and partly due to loop corrections to tree-level processes beyond the NWA in which a leptoquark is exchanged in the $t$-channel (an example of which is shown in figure 9(b)). This latter contribution gives rise to substantial enhancement below resonance. Note however that not all Feynman graphs can be associated unambiguously into one of the two above-mentioned corrections. For example, the graph shown on the r.h.s. of figure 2 can be interpreted as either an NLO correction to the third graph of figure 1 or a non-factorisable correction to the first graph of figure 1 . To highlight these contributions figure 10 makes a comparison between the NWA and nonfactorisable results for a scalar leptoquark by showing the difference between the two sets of results at both LO and NLO. For both the LO and NLO results there are small differences in the resonant region, but the biggest difference is seen in the region $m_{\mathrm{inv}}<m_{\mathrm{LQ}}$ coming 


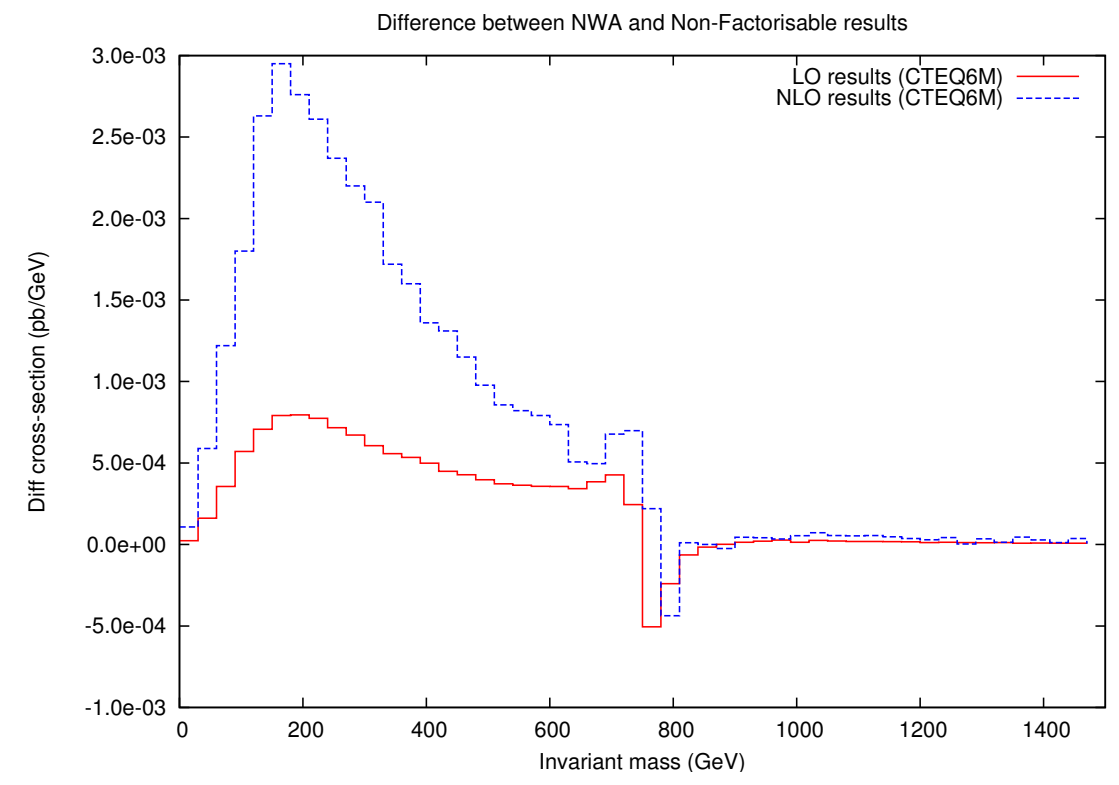

Figure 10. Difference between the NWA and full non-factorisable scalar leptoquark results at LO and NLO.

\begin{tabular}{|l|r|r|}
\hline Correction Type & Peak Height $(\mathrm{pb} / \mathrm{GeV})$ & Percentage change on LO \\
\hline LO & $8.61 \times 10^{-3}$ & - \\
\hline NLO QCD & $1.24 \times 10^{-2}$ & $+44 \%$ \\
\hline NLO Additional & $1.02 \times 10^{-2}$ & $+18 \%$ \\
\hline NLO Total & $1.40 \times 10^{-2}$ & $+62 \%$ \\
\hline
\end{tabular}

Table 3. Summary of the CTEQ6M scalar results for the non-factorisable process.

\begin{tabular}{|l|r|r|}
\hline Correction Type & Total cross-section $(\mathrm{pb})$ & Percentage change on LO \\
\hline LO & 1.08 & - \\
\hline NLO QCD & 1.56 & $+45 \%$ \\
\hline NLO Additional & 1.75 & $+62 \%$ \\
\hline NLO Total & 2.24 & $+107 \%$ \\
\hline
\end{tabular}

Table 4. Summary of the CTEQ6M scalar results for the non-factorisable process.

from the enhancement to the distribution due to the non-factorisable process. The NLO results show the biggest difference in this region which, as discussed, is primarily due to the additional NLO contributions.

\subsection{Renormalization/factorisation scale dependence for scalar leptoquarks}

Performing the calculations at NLO introduces renormalisation and factorisation scales $\mu$ and $\mu_{F}$ respectively. In principal the choice of the values of these scales is arbitrary, however due to the nature of perturbative calculations the NLO corrections will have some sensitivity to $\mu$ and $\mu_{F}$. To determine the sensitivity of the results the NLO cross-section 


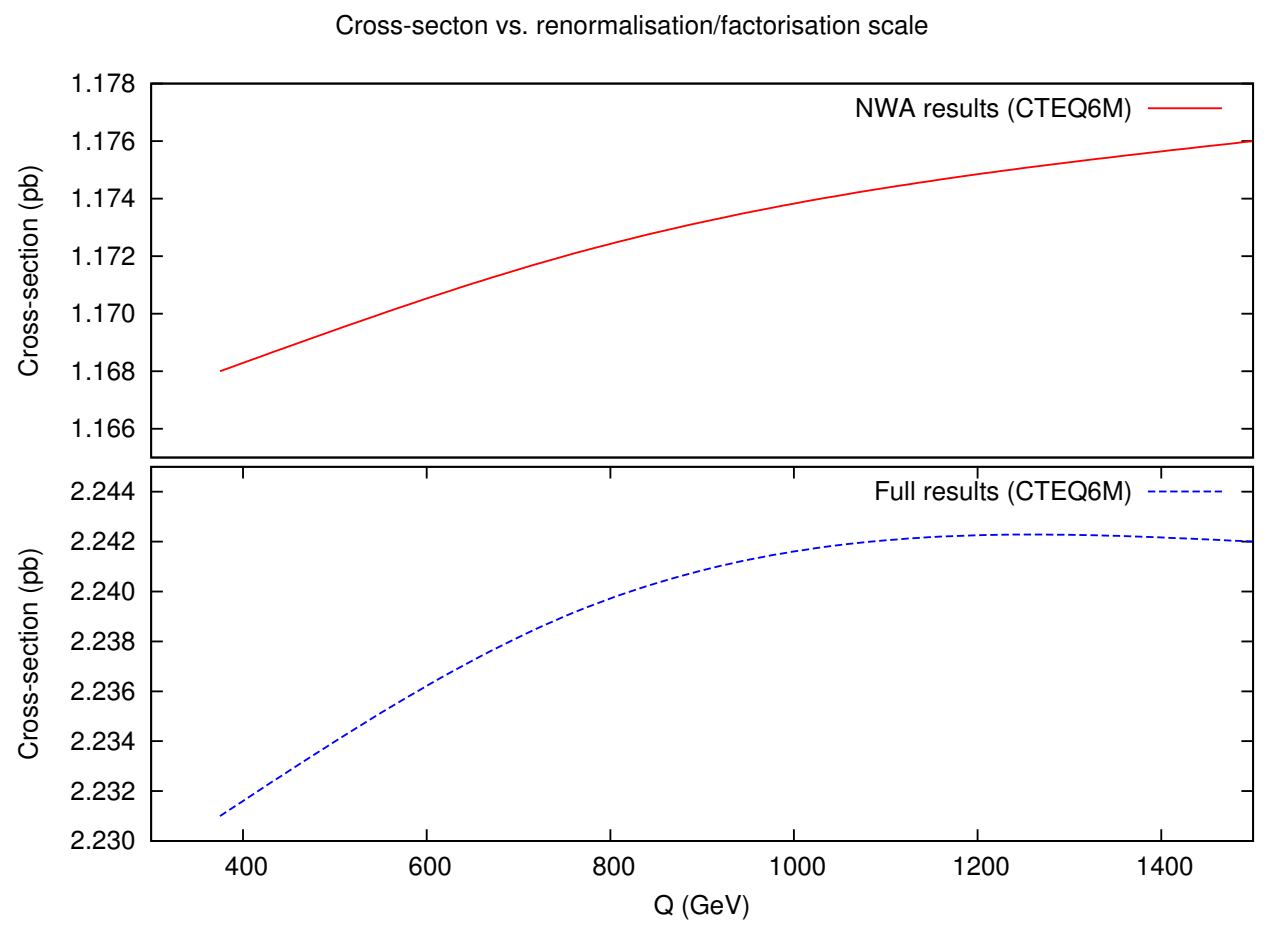

Figure 11. Scale dependence for the NWA and full non-factorisable scalar leptoquark process with the scale $Q=\mu=\mu_{F}$.

has been calculated for a range of different renormalisation and factorisation scales in the range $\left[\frac{1}{2} m_{\mathrm{LQ}}, 2 m_{\mathrm{LQ}}\right]$ where we have limited ourselves to the case in which these two scales are equal.

The results of the scale dependence are given in figure 11 and show that, although there is some scale dependence, the NLO results are reasonably insensitive to a change in $\mu$ and $\mu_{F}$.

\section{$3 \quad$ Vector leptoquarks}

For the study of vector leptoquarks we will consider the $U_{1}$ type leptoquark described in [1]. The core process being studied is

$$
d+g \rightarrow e^{-}+e^{+}+d
$$

and the Feynman diagrams for this process at LO and NLO have the same topologies as in the scalar case. ${ }^{5}$

As before the results are comprised of three main contributions. The LO contributions, the NLO virtual and real QCD corrections to the LO process and the additional NLO tree-level contributions. Again the initial state singularities are handled using the dipole subtraction method.

\footnotetext{
${ }^{5}$ The virtual corrections present one complication due to our choice of gauge for the vector particles: the Feynman gauge. In this gauge contributions from Goldstone bosons and Faddeev-Popov ghosts need to be included with the loop diagrams.
} 


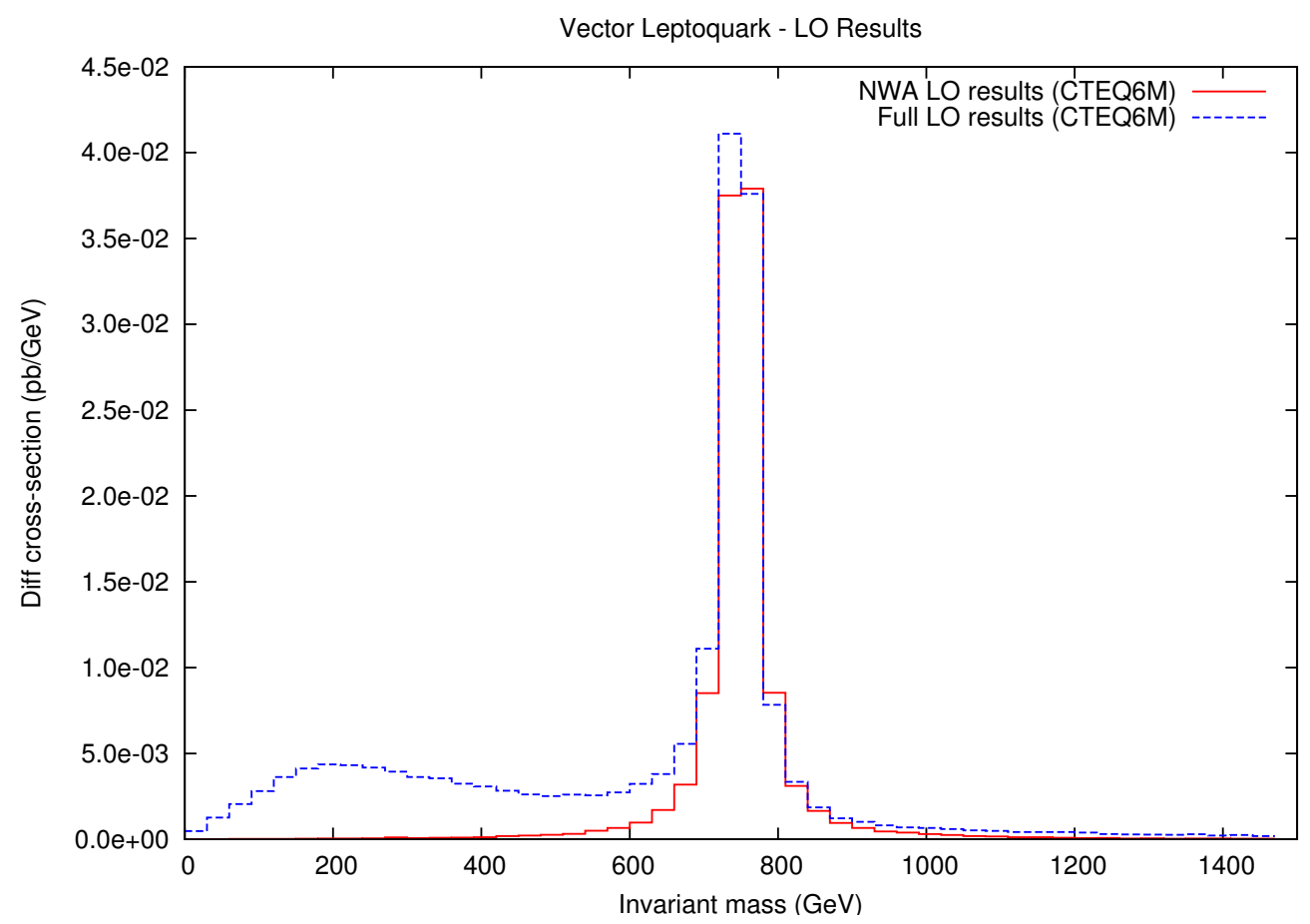

Figure 12. LO results for producing a vector leptoquark - comparing the NWA to the full non-factorisable process (CTEQ6M).

\subsection{NWA results}

The NWA result for the vector leptoquark show the same features as were seen with the scalar leptoquark. The invariant mass distribution is symmetrical with a resonant peak at $m_{\mathrm{LQ}}=750 \mathrm{GeV}$. The $\mathrm{LO}$ result is given in figure 12 and shows a resonant peak height of $3.79 \times 10^{-2} \mathrm{pb} / \mathrm{GeV}$ and comparing this with the NLO result for the core process, (3.1), shown in figure 13, the peak height has increased to $5.23 \times 10^{-2} \mathrm{pb} / \mathrm{GeV}$ giving an enhancement of $38 \%$ over the LO result. There is also an increase in the total cross-section from $3.30 \mathrm{pb}$ to $4.49 \mathrm{pb}$ giving an enhancement of $36 \%$. The K-factor for the NLO QCD contributions shows more variation than with the scalar leptoquark result, but is reasonably constant across the invariant mass distribution with an average value $\sim 1.5$.

The contributions from the additional partonic processes are given in figure 14 and also show a large enhancement to the resonant peak. These corrections have a resonant peak height of $5.39 \times 10^{-2} \mathrm{pb} / \mathrm{GeV}$ giving an enhancement of $42 \%$ over the LO result. There is also a large enhancement to the cross-section which is increased by $44 \%$ to $4.74 \mathrm{pb}$. The $\mathrm{K}$-factor for the additional partonic processes is reasonably constant across the invariant mass distribution with an average value $\sim 1.5$, but shows an enhancement to the K-factor for low values of the invariant mass distribution.

Combining these corrections, the total NLO result is given in figure 15 and shows a peak height of $6.83 \times 10^{-2} \mathrm{pb} / \mathrm{GeV}$ giving a large enhancement of $80 \%$ over the LO result. The cross-section for the total NLO process is increased by $80 \%$ to $5.93 \mathrm{pb}$. The K-factor for the total NLO result is also reasonably constant across the invariant mass distribution 


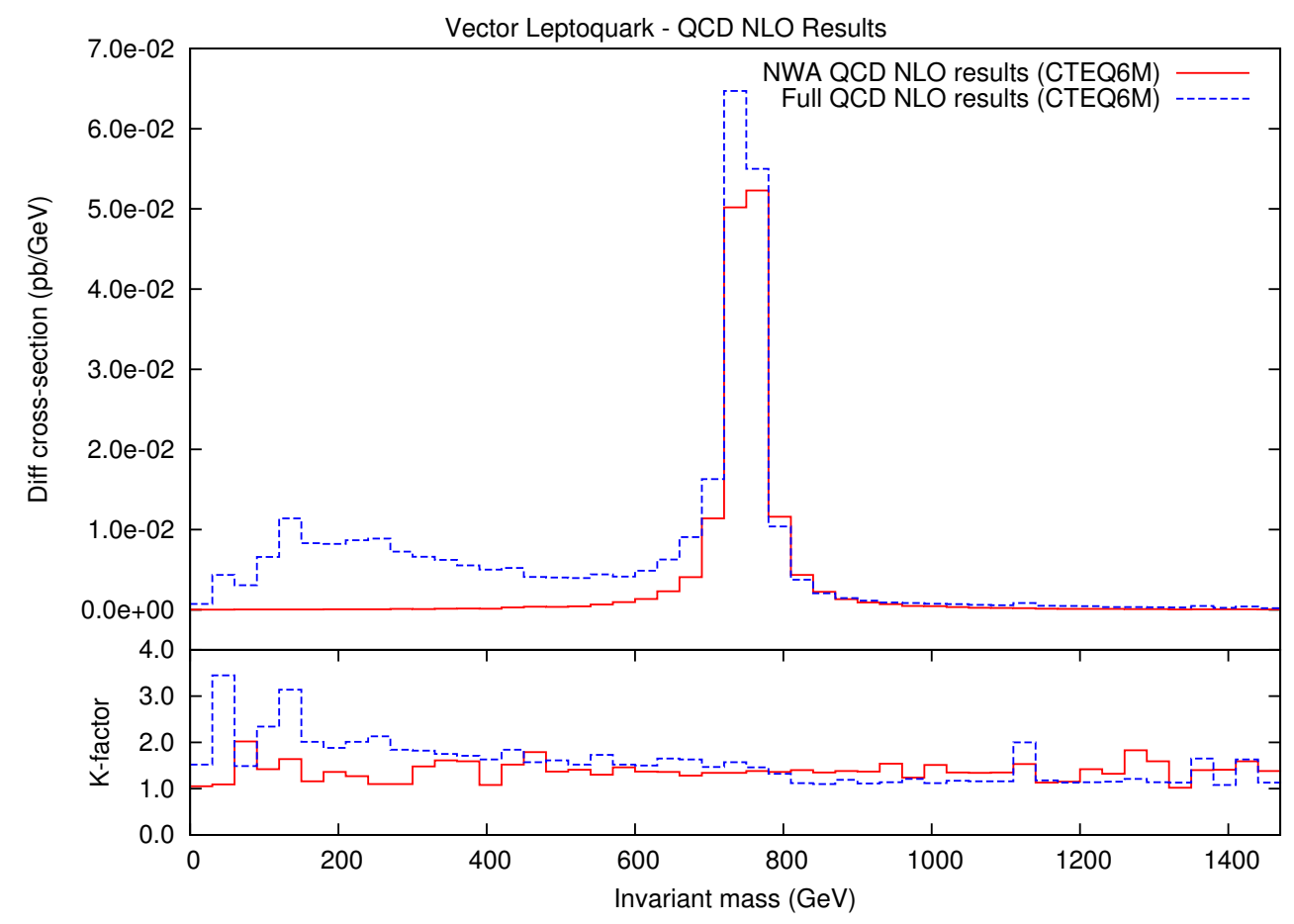

Figure 13. NLO results for producing a vector leptoquark - comparing the NWA to the full non-factorisable core process (CTEQ6M). These results include the QCD corrections only.

\begin{tabular}{|l|r|r|}
\hline Correction Type & Peak Height $(\mathrm{pb} / \mathrm{GeV})$ & Percentage change on LO \\
\hline LO & $3.79 \times 10^{-2}$ & - \\
\hline NLO QCD & $5.23 \times 10^{-2}$ & $+38 \%$ \\
\hline NLO Additional & $5.39 \times 10^{-2}$ & $+42 \%$ \\
\hline NLO Total & $6.83 \times 10^{-2}$ & $+80 \%$ \\
\hline
\end{tabular}

Table 5. Summary of the CTEQ6M vector results for the NWA.

\begin{tabular}{|l|r|r|}
\hline Correction Type & Total cross-section $(\mathrm{pb})$ & Percentage change on LO \\
\hline LO & 3.30 & - \\
\hline NLO QCD & 4.49 & $+36 \%$ \\
\hline NLO Additional & 4.74 & $+44 \%$ \\
\hline NLO Total & 5.93 & $+80 \%$ \\
\hline
\end{tabular}

Table 6. Summary of the CTEQ6M vector results for the NWA.

with an average value $\sim 2$, but does show an increase to the K-factor for low values of the invariant mass distribution, which arises from the contributions from the additional partonic processes. These results are summarised in tables 5 and 6 . 


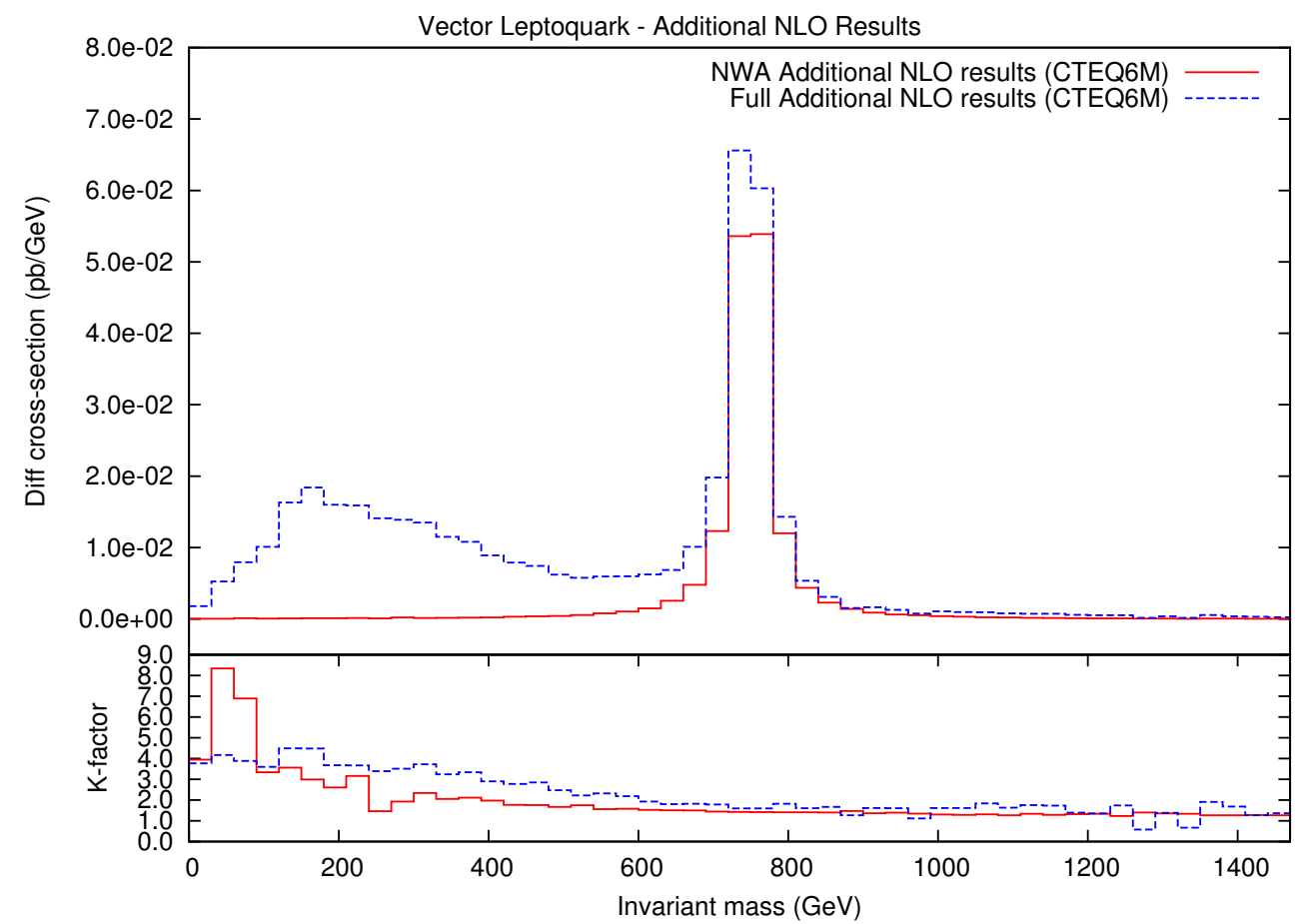

Figure 14. Contributions from the additional partonic processes at NLO for producing a vector leptoquark - comparing the NWA to the full non-factorisable process (CTEQ6M). These results include QCD corrections only.

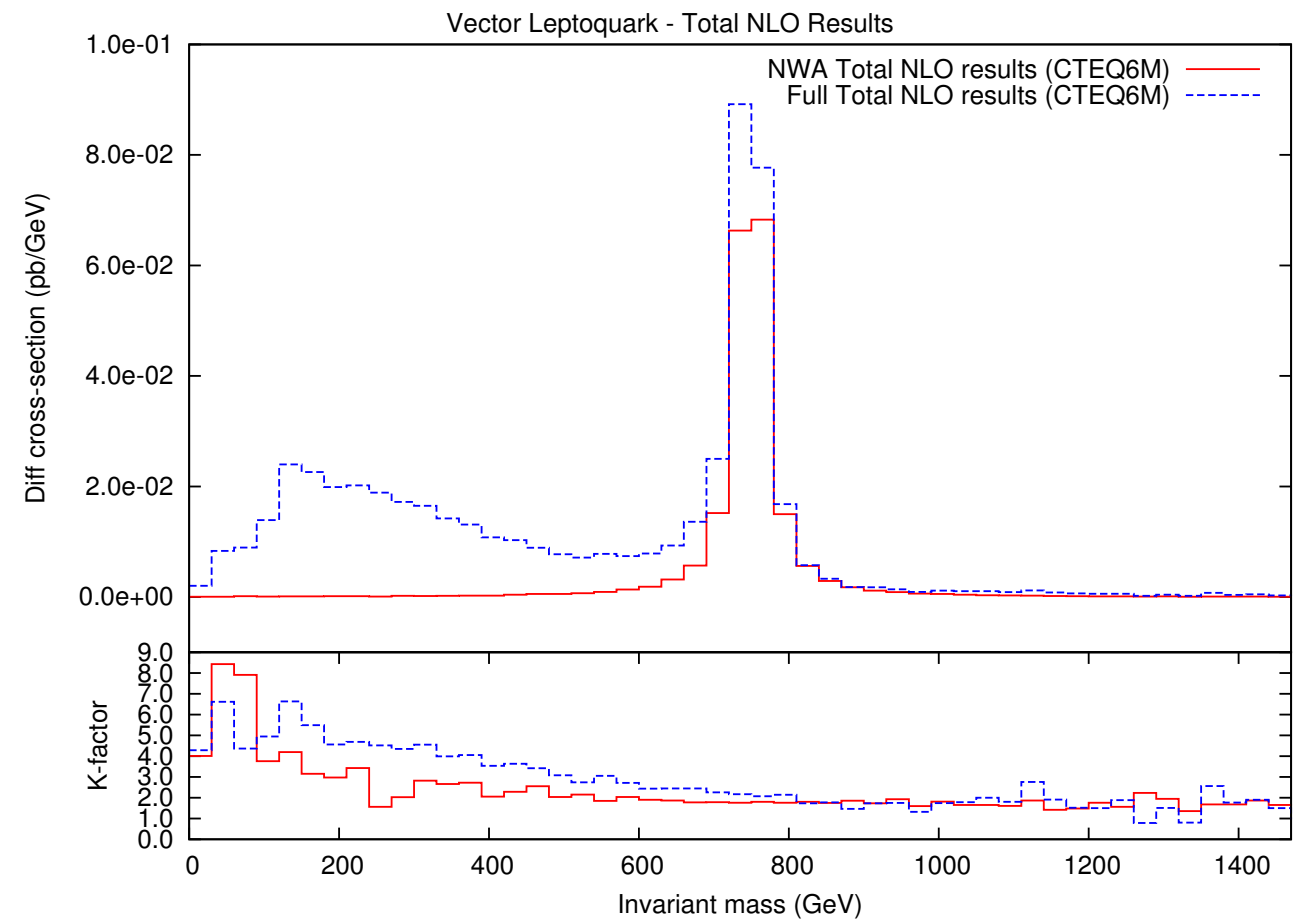

Figure 15. Total NLO results for producing a vector leptoquark - comparing the NWA to the full non-factorisable process (CTEQ6M). 


\subsection{Non-factorisable results}

For the non-factorisable results the $\mathrm{LO}$ contribution is given in figure 12 and shows the same resonant peak at $m_{\mathrm{LQ}}=750 \mathrm{GeV}$ as in the NWA, but compared with the scalar leptoquark results there is now a noticeable difference between the peak heights for the NWA and full process. This is due to the fact that for a vector leptoquark there is interference between amplitudes in which the intermediate leptoquark has different helicities. As in the scalar case the distribution for the full process is not a symmetric and there is an enhancement to the invariant anti-lepton jet invariant mass $m_{\text {inv }}$ for $m_{\text {inv }}<m_{\mathrm{LQ}}$. There is also an increase in the total-cross section, at LO the cross-section for the full non-factorisable process is $5.57 \mathrm{pb}$. This increase is primarily due to the enhancement in the region $m_{\mathrm{inv}}<m_{\mathrm{LQ}}$.

The NLO QCD corrections to the core process, (3.1), are given in figure 13 and show a much bigger enhancement over the LO result than occurs in the NWA. For the full process the peak height is $6.47 \times 10^{-2}$ giving a large enhancement of $57 \%$ over the LO result. The total cross-section also has an increase of $62 \%$ to $9.03 \mathrm{pb}$. The K-factor for the NLO QCD corrections shows some variation, with an increase in the K-factor for low values of the invariant mass distribution, but is otherwise reasonably constant with an average value $\sim 1.5$.

The contributions for the additional partonic processes at NLO, shown in figure 14, also show a much bigger enhancement over the LO result than occurs in the NWA. For the full process the peak height is $6.56 \times 10^{-2} \mathrm{pb} / \mathrm{GeV}$ giving a large enhancement of $60 \%$ over the LO result. The total cross-section also has an enhancement of $121 \%$ to $12.29 \mathrm{pb}$. The K-factor for the additional partonic processes is also reasonably constant across the invariant mass distribution and shows less of an increase for low values of the invariant mass than is apparent in the NWA results.

Combining all of the contributions the total NLO results are shown in figure 15 and has a resonant peak with a height of $8.92 \times 10^{-2} \mathrm{pb} / \mathrm{GeV}$ giving an enhancement of $117 \%$ over the LO result. The cross-section for the total NLO contributions is increased by $183 \%$ to $15.75 \mathrm{pb}$. The K-factor for the total NLO contribution shows an increase for lower values of the invariant mass distribution otherwise is reasonably constant with an average value $\sim 2.0$. A summary of these results is given in tables 7 and 8 .

As with the scalar leptoquarks a comparison between the NWA and non-factorisable results for the vector leptoquark can be made by looking at the differences between the two sets of results, see figure 16. In common with the scalar results there is a difference between the NWA and non-factorisable results in the region $m_{\mathrm{inv}}<m_{\mathrm{LQ}}$ coming from the enhancement to the distribution due to the non-factorisable process. The NLO result again shows the biggest difference which is primarily due to the additional NLO contributions. Compared with the scalar results there are also differences between the vector NWA and non-factorisable results around the resonant peak and in the case of the NLO results this is particularly large. 


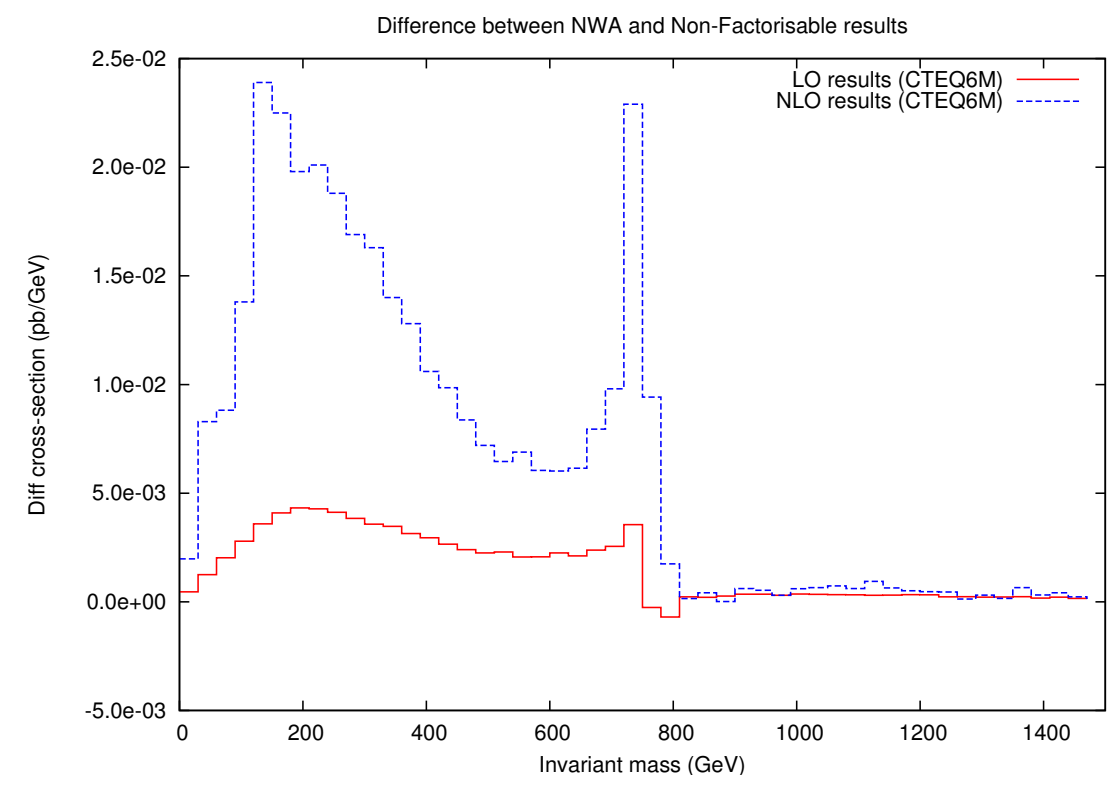

Figure 16. Difference between the NWA and full non-factorisable vector leptoquark results at LO and NLO.

\begin{tabular}{|l|r|r|}
\hline Correction Type & Peak Height $(\mathrm{pb} / \mathrm{GeV})$ & Percentage change on LO \\
\hline LO & $4.11 \times 10^{-2}$ & - \\
\hline NLO QCD & $6.47 \times 10^{-2}$ & $+57 \%$ \\
\hline NLO Additional & $6.56 \times 10^{-2}$ & $+60 \%$ \\
\hline NLO Total & $8.92 \times 10^{-2}$ & $+117 \%$ \\
\hline
\end{tabular}

Table 7. Summary of the CTEQ6M vector results for the non-factorisable process.

\begin{tabular}{|l|r|r|}
\hline Correction Type & Total cross-section $(\mathrm{pb})$ & Percentage change on LO \\
\hline LO & 5.57 & - \\
\hline NLO QCD & 9.03 & $+62 \%$ \\
\hline NLO Additional & 12.29 & $+121 \%$ \\
\hline NLO Total & 15.75 & $+183 \%$ \\
\hline
\end{tabular}

Table 8. Summary of the CTEQ6M vector results for the non-factorisable process.

\subsection{Renormalization/factorization scale dependence for vector leptoquarks}

As with the scalar results the scale dependence for the vector leptoquarks is also checked. The results of the scale dependence for the NWA and full non-factorisable process are shown in figure 17. As in the case with the scalar leptoquarks the NLO results for the vector leptoquarks are also reasonably insensitive to changes in $\mu$ and $\mu_{F}$. 


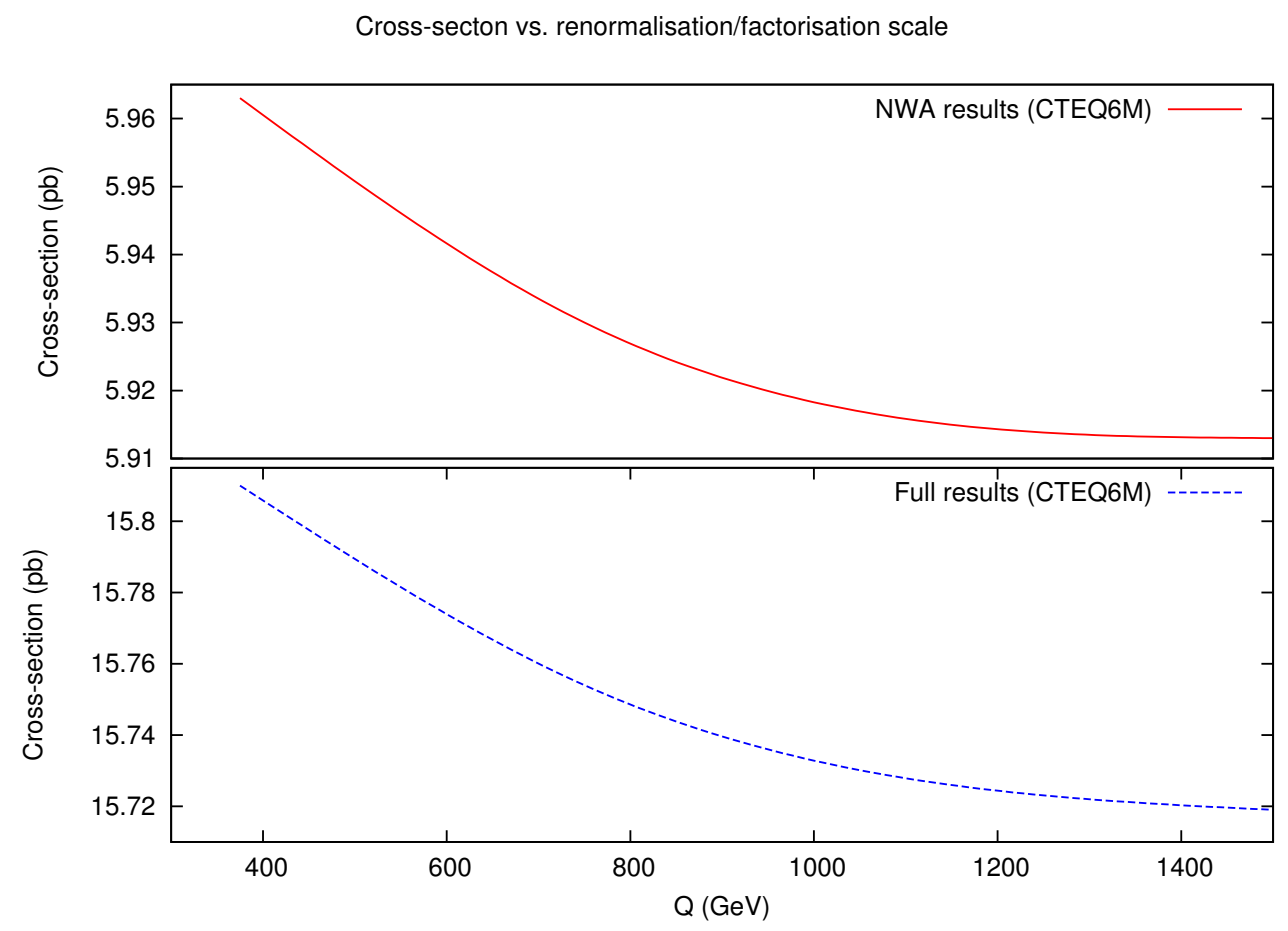

Figure 17. Scale dependence for the NWA and full non-factorisable scalar leptoquark results with the scale $Q=\mu=\mu_{F}$.

\section{Conclusions}

Having calculated the results for the NWA and the full non-factorisable process for both a scalar $\left(R_{2}\right)$ and vector $\left(U_{1}\right)$ leptoquark we will conclude with a discussion and comparison of these results.

\subsection{Scalar leptoquarks}

In general the NWA involving an intermediate scalar particle should give a good approximation to the full result close to the resonant region. The reason for this is there is no sum over helicities to consider and so the only approximation needed is that the decay-width is narrow and the intermediate propagator can be treated as a Dirac $\delta$-function.

Comparing the scalar leptoquark results between the NWA and the full non-factorisable process (see tables 1 and 3) the effectiveness of the NWA approximating the full nonfactorisable process at NLO is confirmed. In particular, we see that both sets of results give good agreement between the heights of the resonant peaks - the dominant feature in both sets of distributions.

Away from the resonant peak there are differences in the invariant mass distributions between the NWA and full non-factorisable process. The NWA gives a symmetric distribution around the resonant peak whereas the non-factorisable process gives a non-symmetric distribution. In particular there is an enhancement to the distribution for values of the invariant mass $m_{\mathrm{inv}}<m_{\mathrm{LQ}}$ coming from the non-factorisable contributions to the full pro- 
cess. The enhancement to the distribution away from the resonant peak does make a large contribution to the total cross-section (see tables 2 and 4) and we see a large difference between the cross-sections calculated in the NWA and full non-factorisable process.

\subsection{Vector leptoquarks}

Comparing the vector leptoquark results the NWA does not provide as good an approximation to the full non-factorisable process for vector leptoquarks as it does for scalars. The key reason for this is because in the NWA the interference between the different helicity states of the intermediate leptoquark are assumed to be negligible and are ignored. Looking at the difference between the resonant peak heights in tables 5 and 7 the agreement between the two sets of results is not as close as in the scalar case and suggests that the helicity interference terms do make a noticeable contribution.

The anti-lepton/jet invariant mass distribution also shows the same features as in the scalar leptoquark case. The NWA gives a symmetric distribution around the resonance, but the full non-factorisable process also shows an enhancement to the distribution for values of the invariant mass $m_{\mathrm{inv}}<m_{\mathrm{LQ}}$. This enhancement makes a sizeable difference to the total cross-section (see tables 6 and 8). The total cross-section for the non-factorisable process is significantly larger than in the NWA and this increase is primarily caused by the additional NLO corrections.

In general when looking at both the scalar and vector leptoquark results there are two important features they have in common:

- The NLO corrections are large compared to the LO results, particularly with regards to the full non-factorisable process, but this is often the case when including QCD corrections.

- From the non-factorisable results there are substantial corrections below the resonance, particularly with regards to the vector leptoquarks, and its is possible these could be observed to give an indirect hint of the presence of leptoquarks.

\section{Acknowledgments}

The authors are grateful to Sasha Belyaev, Mike Seymour and Stefano Moretti for useful conversations and advice. One of us (JBH) wishes to thank STFC for financial support.

Open Access. This article is distributed under the terms of the Creative Commons Attribution License (CC-BY 4.0), which permits any use, distribution and reproduction in any medium, provided the original author(s) and source are credited.

\section{References}

[1] W. Buchmüller, R. Ruckl and D. Wyler, Leptoquarks in Lepton-Quark Collisions, Phys. Lett. B 191 (1987) 442 [Erratum ibid. B 448 (1999) 320] [INSPIRE].

[2] ATLAS collaboration, Search for first generation scalar leptoquarks in pp collisions at $\sqrt{s}=7 \mathrm{TeV}$ with the ATLAS detector, Phys. Lett. B 709 (2012) 158 [Erratum ibid. B 711 (2012) 442] [arXiv: 1112.4828] [INSPIRE]. 
[3] ATLAS collaboration, Search for second generation scalar leptoquarks in pp collisions at $\sqrt{s}=7$ TeV with the ATLAS detector, Eur. Phys. J. C 72 (2012) 2151 [arXiv:1203.3172] [INSPIRE].

[4] ATLAS collaboration, Search for third generation scalar leptoquarks in pp collisions at $\sqrt{s}=7$ TeV with the ATLAS detector, JHEP 06 (2013) 033 [arXiv:1303.0526] [INSPIRE].

[5] CMS collaboration, Search for pair production of first- and second-generation scalar leptoquarks in pp collisions at $\sqrt{s}=7$ TeV, Phys. Rev. D 86 (2012) 052013 [arXiv: 1207.5406] [INSPIRE].

[6] CMS collaboration, Search for Pair-production of Second generation Leptoquarks in 8 TeV proton-proton collisions, CMS-PAS-EXO-12-042 (2013).

[7] CMS collaboration, Search for pair production of third-generation scalar leptoquarks and top squarks in proton-proton collisions at $\sqrt{s}=8 \mathrm{TeV}$, Phys. Lett. B 739 (2014) 229 [arXiv: 1408.0806] [INSPIRE].

[8] A. Belyaev, C. Leroy, R. Mehdiyev and A. Pukhov, Leptoquark single and pair production at LHC with CalcHEP/CompHEP in the complete model, JHEP 09 (2005) 005 [hep-ph/0502067] [INSPIRE].

[9] I. Dorsner, S. Fajfer and A. Greljo, Cornering Scalar Leptoquarks at LHC, JHEP 10 (2014) 154 [arXiv: 1406.4831] [INSPIRE].

[10] T. Mandal, S. Mitra and S. Seth, Single Productions of Colored Particles at the LHC: An Example with Scalar Leptoquarks, JHEP 07 (2015) 028 [arXiv: 1503.04689] [INSPIRE].

[11] M. Krämer, T. Plehn, M. Spira and P.M. Zerwas, Pair production of scalar leptoquarks at the CERN LHC, Phys. Rev. D 71 (2005) 057503 [hep-ph/0411038] [INSPIRE].

[12] M. Krämer, T. Plehn, M. Spira and P.M. Zerwas, Pair production of scalar leptoquarks at the Tevatron, Phys. Rev. Lett. 79 (1997) 341 [hep-ph/9704322] [INSPIRE].

[13] N. Kauer, A threshold-improved narrow-width approximation for BSM physics, JHEP 04 (2008) 055 [arXiv:0708.1161] [INSPIRE].

[14] N. Kauer, Narrow-width approximation limitations, Phys. Lett. B 649 (2007) 413 [hep-ph/0703077] [INSPIRE].

[15] D. Berdine, N. Kauer and D. Rainwater, Breakdown of the Narrow Width Approximation for New Physics, Phys. Rev. Lett. 99 (2007) 111601 [hep-ph/0703058] [INSPIRE].

[16] P. Mastrolia, G. Ossola, T. Reiter and F. Tramontano, Scattering AMplitudes from Unitarity-based Reduction Algorithm at the Integrand-level, JHEP 08 (2010) 080 [arXiv: 1006.0710] [INSPIRE].

[17] S. Catani and M.H. Seymour, A general algorithm for calculating jet cross-sections in NLO QCD, Nucl. Phys. B 485 (1997) 291 [Erratum ibid. B 510 (1998) 503] [hep-ph/9605323] [INSPIRE].

[18] S. Catani, S. Dittmaier, M.H. Seymour and Z. Trócsányi, The dipole formalism for next-to-leading order QCD calculations with massive partons, Nucl. Phys. B 627 (2002) 189 [hep-ph/0201036] [INSPIRE].

[19] T. Plehn, H. Spiesberger, M. Spira and P.M. Zerwas, Formation and decay of scalar leptoquarks/squarks in e p collisions, Z. Phys. C 74 (1997) 611 [hep-ph/9703433] [INSPIRE].

[20] A. Alves, O. Eboli and T. Plehn, Stop lepton associated production at hadron colliders, Phys. Lett. B 558 (2003) 165 [hep-ph/0211441] [INSPIRE]. 